\title{
Why We Mimic Emotions Even When No One is Watching: Limited Visual Contact and Emotional Mimicry
}

\author{
${\text { Michal Olszanowski }{ }^{1} \text {, Monika Wróbel }}^{2}$ \\ ${ }^{1}$ Center for Research on Biological Basis of Social Behavior, SWPS University of Social \\ Sciences \& Humanities in Warsaw, Poland \\ ${ }^{2}$ Institute of Psychology, University of Lodz, Poland
}

Word count: 8110

Corresponding author: Michal Olszanowski, SWPS University of Social Sciences and Humanities, Warsaw, 03-815 Poland, Chodakowska 19/31. E-mail: molszanowski@swps.edu.pl.

Declaration of Conflicting Interests: The authors declared that there were no conflicts of interest with respect to the authorship or the publication of this article.

Author Contributions: M.O. conceptualized and drafted the original idea. M.W. provided critical feedback on the idea and wrote the original manuscript. Both authors contributed to the final version of the manuscript.

Funding: Preparation of this article was supported by grants from the National Science Centre in Poland awarded to Michal Olszanowski [grant number: 2020/37/B/HS6/03538] and Monika Wróbel [grant number: 2017/26/E/HS6/00725]. 


\begin{abstract}
This article explores emotional mimicry and its interpersonal functions under the absence versus presence of visual contact between the interacting partners. We review relevant literature and stress that previous studies on emotional mimicry were focused on imitative responses to facial displays. We also show that the rules applying to mimicking facial expressions may not necessarily be applicable when visual emotional signals are not present (e.g., people attending an online meeting cannot see each other's faces). Overall, our review suggests that emotional mimicry functionally adapts to whether the interacting partners can see each other. We therefore argue that going beyond facial displays may provide insight into emotional mimicry's social functions, thereby clarifying its role in fostering affiliation and emotional understanding.
\end{abstract}

Keywords: emotional mimicry, facial displays, vocal expressions, visual contact, affiliation, emotional understanding 


\section{Why We Mimic Emotions Even When No One is Watching: Limited Visual Contact and Emotional Mimicry}

The growing popularity of videoconferencing has encouraged intense debate on the pros and cons of online meetings. One often-mentioned disadvantage of virtual interactions is that they recreate real-life communication to a limited extent (Bailenson, 2021). Nonverbal cues that are present in face-to-face meetings are often missing, especially when participants have their cameras off and messages sent by facial expressions are not visible. This limited access to visual information may affect interpersonal communication because perception and response to others' facial displays play a crucial role in social interactions (Oh Kruzic et al., 2020). One of the most intriguing phenomena observed during face-to-face interactions is that people show an automatic tendency to mirror each other's facial displays, which is manifested by congruent facial activity and referred to as facial or emotional mimicry (Hess \& Fischer, 2013; Hess, 2021).

Much evidence shows that emotional mimicry serves important social functions by facilitating mutual understanding and affiliation: Mimicking helps the perceiver accurately decode emotional displays of the expresser and increases liking and rapport between them (for reviews, see Hess, 2021; Niedenthal et al., 2017; Kämpf et al., 2018). The majority of previous studies in this area, however, have been focused on emotional mimicry following exposure to facial displays. The question thus arises whether mimicry plays similar functions when visual contact is limited. For instance, will people attending an online meeting be motivated to mimic smiles for affiliative purposes when their cameras are off? Or maybe in this case, mimicry will compensate for the lack of visual information and serve predominantly as a tool to better understand others' internal states? 
In the present article, we take a closer look at emotional mimicry and its social functions under the absence versus presence of visual information. For this aim, we provide a brief overview of recent research on emotional mimicry, with a focus on a few studies on facial mimicry in response to vocal emotional expressions. We also show why the rules that apply to emotional mimicry to facial displays may not necessarily apply to mimicry when there is no visual contact. Finally, we present arguments why studying emotional mimicry under the absence versus presence of visual information may enhance our understanding of what emotional mimicry is and what functions it serves.

\section{Emotional Mimicry as a Social Phenomenon}

The term "mimicry," in its broad sense, refers to the imitation of a variety of others' nonverbal behaviors, including facial expressions (Hess \& Fischer, 2013), gestures (Chartrand \& Bargh, 1999), bodily postures (Bavelas et al., 1986; Bernieri \& Rosenthal, 1991), speech prosody and nonverbal vocalizations (Neumann \& Strack, 2000), or autonomous signals such as heart rate (Feldman et al., 2011) or pupil diameter (Kret et al., 2015). Imitating others' expressed emotions stands out of this category because emotional displays, in contrast to other non-verbal behaviors, are meaningful social signals that inform about the expresser's inner states, intentions, and orientation towards the perceiver (van Kleef, 2017). As a result, the main goal of imitating others' emotional displays is to regulate social interactions (Hess \& Fischer, 2013; Hess, 2021). This unique nature of emotional mimicry is highlighted by recent theories. Most of them hold that mimicry is not a simple reflex-like process based on the perception-action link but a flexible, top-down regulated mechanism that helps to navigate social interactions by serving two interrelated social functions. Specifically, emotional mimicry (1) fosters affiliation by signalizing emotional understanding, and (2) facilitates emotional understanding by providing insightful cues about the expresser's emotional state 
and the meaning of their expressed emotion (Hess \& Fischer, 2013; Hess, 2021; Niedenthal et al., 2005; Winkielman et al., 2018; Wróbel \& Imbir, 2019).

The first of these functions, that is, the role of mimicry in fostering affiliation, is supported by much evidence showing that mimicry increases liking and rapport between interaction partners (for a review, see Hess, 2021). In general, this evidence is consistent with studies on behavioral mimicry, which show that imitating others' gestures, postures, and other motor movements acts as a social glue that bonds individuals together (for a review, see Lakin et al., 2003). The facilitating influence of mimicry on social bonding is attributed to the fact that imitating others communicates attention and emphatic understanding and thus is a strong signal of affiliative intents (Lakin et al., 2003). Accordingly, people tend to mimic others when they are motivated to send affiliative signals, that is, the expresser's intents also seem affiliative (Hess \& Fischer, 2013; Hess, 2021). For instance, mimicry occurs more readily in response to affiliative emotional displays (e.g., happiness) or likeable expressers than antagonizing emotional displays (e.g., anger or disgust) or dislikeable expressers (Fischer et al., 2012). This indicates that the link between mimicry and affiliation is bi-directional: The perceiver's initial liking of the expresser fosters mimicry, and mimicry, in turn, increases the expresser's liking of the perceiver (Kämpf et al., 2018; Likowski et al., 2008).

The second function of emotional mimicry, that is, its role in understanding the emotions of others, remains less clear. The evidence for this role is based on the assumption that mimicking others' expressed emotions provides bodily feedback, which helps the perceiver to re-experience these emotions in him- or herself and, as a result, decode these emotions more accurately (Arnold \& Winkielman, 2020). The rationale underlying this assumption can be found in theories of emotional embodiment, according to which people process emotional information by reactivating neural states associated with their own emotional experiences (Niedenthal, 2007; Winkielman et al., 2018). Therefore, when 
imitating others' emotional expressions, perceivers reactivate corresponding states in themselves, which provides a basis for emotional understanding (Niedenthal, 2007). However, research addressing the effects of imitating others' expressed emotions on the ability to recognize these emotions has yielded an inconsistent pattern of findings, with some studies showing that blocking facial mimicry impairs the recognition of emotions (Oberman et al., 2007; Neal \& Chartrand, 2011; Niedenthal et al., 2001), while others report no relationship between mimicry and emotion recognition accuracy (e.g., Blairy et al., 1999; Hess \& Blairy, 2001). Importantly, research that employs facial blocking, often taken as the evidence for the link between mimicry and emotion recognition, sparked off an intense debate on the reliability of this method (Coles et al., 2019). Moreover, a recent meta-analysis of correlations between facial mimicry and facial emotion recognition provided no support for a significant relationship between the two phenomena (Holland et al., 2021). This suggests that, emotional mimicry may not necessarily serve as a tool to infer emotions from other people's emotional displays (at least under certain conditions, e.g. when the expresser's face is visible).

Notably, the profound role of emotional mimicry in regulating social interactions is highlighted by theories which view mimicry as one of the main mechanisms underlying the transfer of emotions between people, a phenomenon often referred to as emotional contagion (Hatfield, et al., 1994; Prochazkova \& Kret, 2017; Wróbel \& Imbir, 2019). Although some research on the relationship between mimicking and sharing the emotions of others has challenged this view (Hess \& Blairy, 2001; Van der Schalk, et al., 2011), more recent studies have demonstrated that emotional mimicry can indeed be involved in emotional contagion because participants' facial activity in response to emotional displays partially accounts for emotions that participants report later (Olszanowski et al. 2020; Lischetzky, et al., 2020). These studies, however, similar to the majority of studies on emotional mimicry in general, were limited to emotional mimicry and contagion triggered by exposure to facial displays. In 
everyday life, however, the evaluation of an emotional expression is rarely based on facial cues only. More often, we see a face in a specific context, accompanied by a verbal message or vocal expression, or even hear other people talk without seeing them, for instance, when talking on the phone or attending an online meeting with cameras switched off. Although, as we discuss below, the absence of visual information does not eliminate emotional mimicry, the question remains whether the process functionally adapts to emotional signals coming from non-visual sensory channels.

\section{Emotional Mimicry and Limited Visual Contact}

When visual contact is limited, people may glean information about others' emotions from non-facial cues. In such contexts, facial mimicry may likely be replaced by or complemented with vocal mimicry, that is, the imitation of vocal emotional expressions. For instance, people who listen to neutral sentences spoken in an emotional manner spontaneously imitate the expresser's emotional tone of voice (Hatfield et al. 1995; Neuman \& Strack, 2000; Rueff-Lopes et al., 2015). However, vocal mimicry is not always possible because, in some contexts, the perceiver only passively listens to the expresser talk, which precludes the imitation of speech prosody and nonverbal vocalizations. Such contexts leave room for crosschannel mimicry, that is, matching facial responses to non-facial emotional expressions (Hawk \& Fischer, 2016).

A few studies have already demonstrated that congruent facial responses can be elicited by vocal expressions of emotion (Hawk et al., 2012; Hietanen et al., 1998; Lima et al., 2021; Magnée et al., 2007). Specifically, Hietanen and colleagues (1998) showed that a vocal expression of anger evoked more intense responses of corrugator supercilii (i.e., the "frowning muscle") and less intense responses of orbicularis oculi (i.e., the "crow's feet muscle" typically associated with authentic smiles) than a vocal expression of contentment. In a related vein, Magnee et al. (2007) found that congruent combinations of facial and vocal 
expressions of fear increased corrugator activity, while congruent combinations of facial and vocal expressions of happiness increased zygomaticus activity (the "smiling muscle"). Importantly, no increase in zygomaticus and corrugator activity was observed when participants were exposed to incongruent combinations of facial and vocal expressions (that is, a fearful face was combined with a happy voice or a happy face was combined with a fearful voice), which suggests that both of these channels were important for triggering a mimicry response. Arias et al. (2018) used digitally transformed sentences that sounded as if they were spoken with a smile on the speaker's face and found that participants who listened to these sentences responded to them with increased zygomaticus activity. Hawk et al. (2012) demonstrated that hearing and repeating vocalizations of anger, disgust, sadness, and happiness evoked corresponding facial expressions and self-reported emotions in the perceiver. Further and even more importantly, the inhibition of participants' facial activity impaired their ability to process emotional vocalizations and reduced participants' subjective experience of corresponding emotional states. Finally, Lima et al. (2021) examined facial electromyographic responses to nonverbal vocalizations and found that authentic laughs, as compared to posed laughs, evoked stronger activity of the muscles associated with positive affect (zygomaticus and orbicularis). Additionally, more intense orbicularis activity predicted higher perceived laughter authenticity, whereas more intense corrugator activity predicted lower perceived laughter authenticity, which suggests that facial responses were related to how participants interpreted vocal expressions they were exposed to.

Taken together, these findings show that vocally expressed emotions evoke congruent facial muscle activity in perceivers, thereby suggesting that, despite rather modest empirical interest in cross-channel mimicry, facial responses to vocal expressions of emotion are remarkably similar to mimicry of facial expressions. Moreover, Hawk et al.'s (2012), as well as Lima et al.'s (2021) studies, indicate that cross-modal mimicry may contribute to emotional 
understanding in a similar way unimodal mimicry does. This implies that both types of imitation - in response to facial and vocal emotional displays - should be equally important in regulating social interactions (see also Hawk \& Fischer, 2016). However, some indirect evidence challenges the notion that facial mimicry to vocal displays and facial mimicry to facial displays are functionally the same. More specifically, exposure to the vocal displays of emotion eliminates one important feature of social interaction, namely, the experience of being watched. This experience is an important factor in eliciting facial responses - for instance, when people are aware that they are being watched, they are more likely to send affiliative signals (e.g., respond with affiliative smiles; Hietanen \& Peltola, 2021).

Does it mean that mimicry and its functions can be contextually regulated by factors such as whether the perceiver can see the expresser's face or whether the expresser can see the perceiver's response? Or, put differently, does limited access to visual information modulate the extent to which mimicry is functionally used as a tool for affiliation and understanding of the expresser's internal state? Getting back to the example of an online meeting with cameras turned off, the perceiver may have much less motivation to signal affiliation when the expresser cannot see them. In this case, mimicry may be more likely to be used as bodily feedback to better understand the expresser's internal state and compensate for the absence of visual information. Accordingly, it is possible that emotional mimicry serves different functions depending on whether or not interaction partners can see each other. Below, we review and discuss evidence supporting this claim. We also argue that going beyond the imitation of facial displays and testing participants' responses to various combinations of facial and vocal expressions may help clarify the role of emotional mimicry in affiliation and understanding of others' internal states. 


\section{Visual Contact and Emotional Mimicry as a Tool for Fostering Affiliation}

As already stated, facial responses to vocal emotional displays (under the absence of visual contact) resemble imitative responses to facial displays (Hawk \& Fischer, 2016). At the same time, visual contact seems to be an important factor activating (or at least facilitating) the affiliative function of emotional mimicry. Specifically, as mentioned above, it is possible that people are more motivated to mimic others for affiliative purposes when they are aware that those others are able to pick up the affiliative signals they sent. Hence, it seems reasonable to assume that mimicking others to build liking and rapport may be more likely to occur when the perceiver is aware that the expresser is able to see their imitative responses.

This reasoning is indirectly supported by studies showing that both behavioral and emotional mimicry is modulated by eye gaze direction. For instance, direct (as opposed to averted) eye gaze enhances mimicry of hand movements (Wang et al., 2011; Wang \& Hamilton, 2014). A similar pattern of results has been found for emotional mimicry in response to happy faces, that is, direct gaze was shown to evoke higher zygomaticus activity following exposure to happy expressions than averted gaze (Kuang et al., 2021; Marschner et al., 2015; Rychlowska et al., 2012; Schrammel et al., 2009; Soussignan et al., 2013). When it comes to emotional mimicry to less affiliative emotional displays (i.e., angry faces), the findings are less consistent, with some studies showing that direct gaze evokes higher corrugator activity than averted gaze (Schrammel et al., 2009; Soussignan et al., 2013), while other studies report no such effect (Kuang et al., 2021; Marschner et al., 2015).

The fact that eye gaze direction consistently modulates responses to faces expressing affiliative emotions suggests that eye contact may be particularly important when it comes to the affiliative function of emotional mimicry. In general, emotional faces with direct gaze signal liking and affiliation, while emotional faces with averted gaze signal social exclusion and aversion (Leng et al., 2018; Mason et al., 2005). Therefore, given that mimicry is more 
likely to occur when the goal to affiliate is activated (Hess, 2021; Hess \& Fischer, 2014), the positive influence of direct eye contact on mimicry responses seems a logical consequence of mimicry's affiliative function. Still, the question remains whether it is the direct eye contact itself or rather the awareness of being seen by the expresser that fosters imitative responses.

An indirect answer to this question may be found in studies showing that the awareness of being watched is a strong moderator of perceivers' responses to faces with direct vs averted gaze (Jarick \& Bencic, 2019; Hietanen et al., 2020; Myllyneva \& Hietanen, 2015). For instance, Myllyneva and Hietanen (2015) showed that greater skin conductance, heart rate, and cortical $\mathrm{P} 3$ responses to direct vs averted gaze were observed only when participants were led to believe that another person was able to see them through an electronic shutter. When participants believed that this person was unable to see them, no effect of gaze direction on participants' reactions was found. In a similar vein, Jarick and Bencic (2019) demonstrated that autonomic arousal, as indicated by skin conductance, was enhanced when interaction partners made direct eye contact, that is, turned their heads towards each other (in contrast to turning their heads away). This, however, was evident only when the contact was possible; once gaze signals became obscured or blocked (i.e., participants wore sunglasses or were blindfolded, respectively), arousal significantly decreased and was no longer modulated by sending or receiving direct gaze.

Even more compelling evidence comes from a recent study by Hietanen and Peltola (2021). Based on the observation that eye contact often elicits smiles in perceivers, the authors investigated whether a perceiver's awareness that another person is able to see their direct gaze would influence their smiling responses. Participants wore clear or dark lens glasses, which made the other person able or unable to see their eyes, respectively. The results showed that participants' zygomatic responses were more intense when they were aware that their eyes were visible. This suggests that the awareness that another person is able to pick up 
affiliative signals significantly strengthened participants tendency to behave in an affiliative way (i.e., react with smiles).

Does it mean that facial mimicry is also fostered by the awareness of being watched?

On the one hand, the aforementioned studies indicating that direct eye contact increases happiness mimicry (Kuang et al., 2021; Marschner et al., 2015; Rychlowska et al., 2012; Schrammel et al., 2009; Soussignan et al., 2013) suggest that mimicry may be stronger due to the feeling of being monitored by the expresser. On the other hand, given that direct eye contact is not only a signal of watching someone but also an affiliative cue indicating approach tendencies (Leng et al., 2018; Mason et al., 2005), it is possible that faces with direct gaze (relative to faces with an averted gaze) may be more likely to evoke mimicry due to the affiliative signals they send rather than due to simply informing the perceiver that he/she is being watched by the expresser. In fact, the only study we are aware of that directly tested the influence of the awareness of being watched on facial mimicry led to inconclusive findings. Specifically, Hietanen et al. (2019) measured participants' zygomatic and corrugator responses to smiling and neutral faces and manipulated participants' awareness of being watched by using an electronic shutter. The results revealed that the difference in zygomatic activity following exposure to smiling vs neutral faces was more pronounced when participants believed they were being watched by the expresser (as compared to when they believed they were not being watched). This pattern, however, was mainly influenced by participants' responses to neutral rather than smiling faces. Specifically, zygomaticus response to a neutral face was inhibited when participants believed that they were being watched, whereas zygomaticus response to a smiling face was not modulated by this belief. These findings, according to the authors, may mean that smiling in response to another person's smile is a strong automatic reaction that is relatively independent of whether the perceiver believes that they are being watched by the expresser. 
Overall, current evidence does not explain whether the absence of visual contact reduces emotional mimicry, and if so, whether this reduction is caused by the lack of affiliative signals typically sent via face (or eye contact, in particular) or by the perceiver's awareness that their imitative responses are not visible to the expresser. Therefore, future research is needed to further examine the role of facial mimicry when there is two-way, oneway, or the lack of visual contact.

\section{Visual Contact and Emotional Mimicry as a Tool for Facilitating Emotional \\ Understanding}

As already mentioned, the evidence for the second function of emotional mimicry, that is, its role in emotional understanding is largely inconsistent, with many studies showing no support for the link between facial mimicry and facial emotion recognition (for a metaanalysis, see Holland et al., 2021). We should note, however, that the number of studies that examined the role of emotional mimicry for emotion recognition accuracy is low, and most of these studies were focused on a narrow set of prototypical emotional expressions (Holland et al., 2019). Such prototypical emotional expressions (i.e., stereotypical configurations of facial movements indicative of so-called basic emotions, Ortony \&Turner, 1990) differ from spontaneous, multimodal emotional expressions encountered in real life (Barrett et al., 2019; Israelashvili et al., 2019). Most importantly, they are often much easier to decode than natural, more complex emotional expressions (Fridenson-Hayo et al., 2016; Krumhuber et al., 2019). Moreover, the recognition of real-life expressions is based on combined signals from various sources such as facial displays, vocal expressions, body postures, and verbal content. As a result, real-life emotional expressions are often ambiguous and susceptible to the influence of contextual information (Hassin et al., 2013). Accordingly, the null effects of emotional mimicry on basic emotion recognition accuracy observed in previous studies may stem from the fact that mimicking is less useful when the emotion categorization task is relatively easy 
to perform. These null results, however, may not preclude the usefulness of emotional mimicry when more complex, real-life emotional expressions are being decoded.

Notably, the idea that emotional mimicry plays a crucial role in emotional understanding when emotional information is relatively difficult to decode is often mentioned in the context of the Simulation of Smiles (SIMS) model (Niedenthal et al., 2010). The model holds that smiles are not unequivocal signals of joy (as suggested by the basic emotion account), but their social meaning is much more complex and dependent on the circumstances in which smiles appear. As a result, differentiating between different types of smiles (for instance, reward and affiliative smiles) is far more difficult than a simple categorization of a smile as such and thus, may require the involvement of emotional mimicry. In line with this assumption, a recent study by Orlowska et al. (2021) has demonstrated that, when verbal contextual information suggested that the affiliative smile appeared in a reward-related context, participants qualified such a smile as a happy (reward) smile, which indicates that they did not recognize it correctly. However, of importance here, this effect was more pronounced when participants' mimicry was restricted. For participants who could freely mimic the observed smiles, this confusing influence of verbal contextual information on the smile interpretation was reduced. Put another way, restricting emotional mimicry made smile discrimination more difficult and thus increased participants' reliance on additional cues (i.e., verbally presented contextual information).

Taken together, these results suggest that emotional mimicry may be much more useful when the recognition and interpretation of others' emotions is challenging. We think that this applies in particular to situations in which people do not see others' expressed emotions and thus need to recognize and understand these emotions on the basis of limited information. For instance, situations such as talking on the phone or participating in an online meeting where participants have their cameras turned off make emotional recognition more 
difficult than solving a standard basic emotion recognition task and therefore, may call for the engagement of additional tools, such as emotional mimicry.

It is also worth noting that the role of each modality varies in the processing of emotional stimuli. In general, people preferentially categorize emotion expressions based on visual modality, which demonstrates visual dominance in emotional processing (Collignon et al., 2008; Paulmann \& Pell, 2011). However, integrated auditory and visual emotional signals facilitate emotional recognition (Paulmann \& Pell, 2011), and information perceived through one sensory channel can alter information processing in another channel (de Gelder \& Vroomen, 2000). Further, when the availability of visual stimuli is reduced, categorization can be made through the auditory modality (Collignon et al., 2008), which supports the notion that when visual information is not available people focus on vocal cues. Yet, given that, in general, categorizing emotional expressions based on vocal modality is more difficult than when visual modality is involved (Collignon et al., 2008; Paulmann \& Pell, 2011), it seems plausible that the absence of visual cues and the focus on vocal cues may trigger the second function of emotional mimicry, that is, its role in emotional understanding.

This reasoning is indirectly backed up by the above-mentioned studies on how facial mimicry in response to vocal expressions affects emotion processing. For instance, as already mentioned, Lima et al. (2021) showed that participants' facial responses were related to how they assessed the authenticity of spontaneous and posed laughs (which were presented via audio channel). Notably, given that people are not very accurate at differentiating genuine and fake emotional expressions (Ekman \& O’Sullivan, 1991; Hess \& Kleck, 1994), it is reasonable to assume that such a task was difficult, which possibly explains why emotional mimicry was of help here. Similarly, Hawk et al. (2012) demonstrated that inhibiting facial mimicry limited the processing of vocal emotional expressions. More specifically, participants were exposed to audio recordings of two contrasting emotion vocalizations - 
laughing and crying (in a counterbalanced order) - in which an initial sound gradually decreased in volume while the second sound gradually increased in volume (so that both sounds overlapped for a short period). Participants' task was to identify the moment at which the sounds of laughing and crying overlapped. The results showed that participants who could freely move their facial muscles were slower to respond to the shift between the sounds than participants whose mimicry was restricted. This, according to the authors, suggests that facial mimicry facilitated a stronger focus on the first sound, thereby resulting in ignoring the second (incongruent) sound. Put differently, facial mimicry promoted deeper emotional processing of the first sound, which provides support for the role of cross-channel mimicking in emotion understanding.

Summing up, current evidence indirectly shows that limited visual contact between the interacting partners may activate the second function of emotional mimicry, that is, its role in emotional understanding. We thus believe that future research could benefit from investigating the functions of emotional mimicry under the absence vs presence of visual contact and we provide arguments for this claim below.

\section{Visual Contact, Emotional Mimicry, and the Social Meaning of the Expressed Emotion}

In the previous sections, we have analyzed the social functions of emotional mimicry under the absence versus presence of visual contact without regard to the social meaning of the expressed emotion. Such meaning, however, may have a considerable impact on triggering mimicry's social functions, and, as we argue below, this impact may be modulated by the absence vs. presence of visual information.

The idea that emotional expressions bear social meaning is rooted in the social functional approach to emotions, which holds that emotions are social signals that may either strengthen or weaken relationships with others (Fischer \& Manstead, 2008). This assumption seems particularly important to understand the affiliative function of emotional mimicry, 
which - as already mentioned - is activated when the perceiver's goal is to affiliate with the expresser (Hess \& Fischer, 2014). Specifically, research on facial mimicry in response to facial emotional expressions shows that people tend to mimic intrinsically affiliative facial displays (e.g. smiles) but avoid imitating non-affiliative facial displays (e.g. frowns or sneers; for reviews, see Hess \& Fischer, 2013, 2014). This suggests that when visual contact is present (that is, the perceiver is aware that the expresser can see their facial responses), the imitation strategy is set to, paraphrasing Bing Crosby's song, "accentuate the positive and eliminate the negative", which means an increased smiling response to affiliate and a decreased frowning response to avoid antagonism.

The situation changes when visual contact is limited. Here, mimicry of negative emotions does not increase the risk of signalizing antagonism because the expresser is not able to pick up the signals sent via the perceiver's facial responses. As such, the lack of visual contact may boost the role of emotional mimicry in emotion recognition, which should happen in particular when the perceiver is motivated to understand the expresser's inner feelings. In such a case, it is possible that people will imitate not only affiliative emotional displays but also non-affiliative emotional displays, as this may help them gain insight into the expresser's intentions and orientation towards them.

It is also worth adding that the strategic use of emotional mimicry as a tool that facilitates emotional understanding may depend on the emotion being expressed because some emotions may be particularly difficult to recognize when presented via a vocal channel. For instance, when exposed to vocal expressions, people are more accurate in decoding anger and sadness relative to fear and happiness (Juslin \& Laukka, 2003; Hawk et al., 2009). Interestingly, this pattern is different for facial displays, where the highest accuracy rates are observed for happiness, whereas fear and surprise are often confused (Hawk et al., 2009; Olszanowski et al., 2015). 
In short, current literature suggests that both functions of emotional mimicry may be differently activated by different emotions depending on these emotions' affiliative vs. nonaffiliative social meaning. This activation, however, seems to be additionally modulated by whether or not the interaction partners can see each other, which provides further (yet still indirect) evidence for the notion that emotional mimicry strategically adapts to a given situation in order to foster affiliation or facilitate emotional understanding.

\section{Future Directions in Research on Emotional Mimicry Under the Absence vs Presence of}

\section{Visual Information}

Overall, the reviewed literature provides some initial support for the notion that the functions of emotional mimicry may contextually adapt to whether the perceiver can see the expresser's face and whether the expresser can see the perceiver's imitative response. This suggests that visual contact may be important for triggering mimicry's affiliative function whereas the lack of such contact may foster mimicking for emotional understanding purposes. However, the support for this claim remains largely indirect and future research is needed to test it directly. Importantly, such research should provide insight into the mechanisms behind the role of visual contact for using mimicry as a tool for affiliation or understanding of the expresser's internal state because these mechanisms remain unclear. For instance, when the transfer of affiliative information through the visual channel is blocked (e.g., the perceiver cannot see the expresser's affiliative facial display), it is difficult to tell whether mimicry is reduced because affiliative signals sent by the expresser's face are not transferred to the perceiver or because the perceiver realizes that their imitative responses are not visible to the expresser and thus there is no point in mimicking.

We believe that using an alternative means to transfer affiliative vs non-affiliative information (e.g., a happy or angry vocal expressions, respectively) may shed light on these mechanisms. More specifically, testing participants responses not only to facial but also to 
vocal emotional displays may clarify how emotional mimicry functionally adapts to whether or not interaction partners can see each other. If it turns out that people are equally likely to mimic others' expressed emotions regardless of whether affiliative information is transferred only via a vocal channel or both via vocal and facial channels, this would mean that the experience of being watched is not necessary for emotional mimicry to occur and the role of mimicry is not limited to sending affiliative signals. In such a case mimicry will predominantly serve the purpose of understanding others' expressed emotions.

The research testing and comparing both joint and separate effects of emotional signals sent via visual and vocal channels on emotional mimicry may help fill in an important gap in the literature. To the best of our knowledge, only a few studies we mentioned above have attempted to determine the relationship between the auditory perception of emotions and facial mimicry. These studies, however, where either limited to the expressions presented via one sensory channel, or focused on the coherence between participants' responses to facial and vocal expressions. It is thus our understanding that further studies on the role of emotional mimicry in response to combined facial and vocal expressions are necessary. First, such studies are more ecologically valid because the rely on stimuli that resemble everyday multimodal emotional expressions to a greater extent than unimodal emotional displays. Second, given the growing popularity of remote communication in today's world, it is crucial to understand the role of emotional mimicry when visual contact is limited.

We should also note that future studies on the role of emotional mimicry under the absence vs. presence of visual contact would benefit from including emotional displays of different emotions. As already stated, different emotional expression vary in (1) the extent to which they convey affiliative vs. non-affiliative information, and (2) how easily they are recognized via vocal and visual channels. Therefore, the inclusion of various emotional 
displays may shed light on the mimicry's social functions when visual contact is present or absent.

\section{Conclusion}

Despite huge interest in emotional mimicry, most studies in this area were focused on mimicking facial displays. Here, we argue that research addressing the imitation of other (e.g. vocal) emotional expressions, while still being in its infancy, may be crucial for understanding whether emotional mimicry functionally adapts to the absence vs presence of visual contact between the interacting partners.

We believe that getting insight into how the functions of emotional mimicry are modulated under different circumstances may clarify the role of this process in fostering affiliation and understanding of others' internal states. Over the years, these two functions of emotional mimicry have been accentuated somewhat differently by different theoretical approaches. Generally speaking, the embodied cognition theories have primarily emphasized the influence of mimicry on emotional understanding (e.g., Arnold \& Winkielman, 2020; Winkielman et al., 2018), while more socially oriented models have focused on mimicry’s affiliative role in human interaction (Fischer \& Hess, 2017; Hess, 2019). We believe that examining the role of facial mimicry when there is two-way, one-way, or the lack of visual contact may bridge the gap between these two frameworks, by showing that the realization of these two social functions does not occur rigidly but follows flexible situation-dependent rules. 


\section{References}

Arias, P., Belin, P., \& Aucouturier, J.-J. (2018). Auditory smiles trigger unconscious facial imitation. Current Biology, 28, 782-783. https://doi.org/10.1016/j.cub.2018.05.084

Arnold, A. J., \&Winkielman, P. (2020). The mimicry among us: Intra- and inter-personal mechanisms of spontaneous mimicry. Journal Nonverbal Behavior, 44, 195-212. https://doi.org/10.1007/s10919-019-00324-z

Bailenson, J. N. (2021). Nonverbal overload: A theoretical argument for the causes of Zoom fatigue. Technology, Mind, and Behavior, 2. https://doi.org/10.1037/tmb0000030

Barrett, L. F., Adolphs, R., Martinez, A., Marsella, S., \& Pollak, S. (2019). Emotional expressions reconsidered: Challenges to inferring emotion in human facial movements. Psychological Science in the Public Interest, 20, 1-68.

\section{https://doi.org/10.1177/1529100619832930}

Bavelas, J. B., Black, A., Lemery, C. R., \& Mullet, J. (1986). “I show how you feel”: Motor mimicry as a communicative act. Journal of Personality and Social Psychology, 50, 322-329. https://doi.org/10.1037/0022-3514.50.2.322

Bernieri, F. J., \& Rosenthal, R. (1991). Interpersonal coordination: Behavior matching and interactional synchrony. In R. S. Feldman \& B. Rimé (Eds.), Fundamentals of Nonverbal Behavior (pp. 401-432). New York: Cambridge University Press

Blairy, S., Herrera, P., \& Hess, U. (1999). Mimicry and the judgement of emotional facial expressions. Journal of Nonverbal Behavior, 23, 5-41. https://doi.org/10.1023/A:1021370825283

Chartrand, T. L., \& Bargh, J. A. (1999). The chameleon effect: The perception-behavior link and social interaction. Journal of Personality and Social Psychology, 76, 893-910. https://doi.org/10.1037/0022-3514.76.6.893 
Coles, N. A., Larsen, J. T., \& Lench, H. C. (2019). A meta-analysis of the facial feedback literature: Effects of facial feedback on emotional experience are small and variable. Psychological Bulletin, 145, 610-651. https://doi.org/10.1037/bul0000194

Collignon, O., Girard, S., Gosselin, F., Roy, S., Saint-Amour, D., Lassonde, M., \& Lepore, F. (2008). Audio-visual integration of emotion expression. Brain Research, 1242, 126-

\section{5. https://doi.org/10.1016/j.brainres.2008.04.023}

De Gelder, B., \& Vroomen, J. (2000). The perception of emotions by ear and by eye. Cognition \& Emotion, 14, 289-311. https://doi.org/10.1080/026999300378824

Ekman, P., \& O’Sullivan, M. (1991). American Psychologist, 46, 913-920. https://doi.org/10.1037/0003-066X.46.9.913

Farley, S. D. (2021). Introduction to the special issue on emotional expression beyond the face: On the importance of multiple channels of communication and context. Journal of Nonverbal Behavior. https://doi.org/10.1007/s10919-021-00377-z

Feldman, R., Magori-Cohen, R., Galili, G., Singer, M., \& Louzoun, Y. (2011). Mother and infant coordinate heart rhythms through episodes of interaction synchrony. Infant Behavior \& Development, 34, 569-577. https://doi.org/10.1016/j.infbeh.2011.06.008

Fischer, A. H., Becker, D., \& Veenstra, L. (2012). Emotional mimicry in social context: the case of disgust and pride. Frontiers in Psychology, 3, 475.

https://doi.org/10.3389/fpsyg.2012.00475

Fischer, A. H., \& Manstead, A. S. R. (2008). Social functions of emotion and emotion regulation. In M. Lewis, J. Haviland, \& L. Feldman Barrett (Eds.), Handbook of Emotion (3rd edition) (pp. 456-468). New York, NY: Guilford Press.

Fridenson-Hayo, S., Berggren, S., Lassalle, A., Tal, S., Pigat, D., Bölte, S., Baron-Cohen, S., \& Golan, O. (2016). Basic and complex emotion recognition in children with autism: 
Cross-cultural findings. Molecular Autism, 7, 52. https://doi.org/10.1186/s13229-016$\underline{0113-9}$

Hassin, R. R., Aviezer, H., \& Bentin, S. (2013). Inherently ambiguous: Facial expressions of emotions in Context. Emotion Review, 5, 60-65.

https://doi.org/10.1177/1754073912451331

Hatfield, E., Hsee, C. K., Costello, J., Weisman, M. S., \& Denney, C. (1995). The impact of vocal feedback on emotional experience and expression. Journal of Social Behavior \& Personality, 10, 293-312.

Hawk, S., \& Fischer, A. H. (2016). More than just a mirror: Examining the cross-channel mimicry of emotional expressions. In U. Hess \& A. Fischer (Eds.), Emotional mimicry in social context (pp. 107-124). Cambridge University Press. https://doi.org/10.1017/CBO9781107587595.007

Hawk, S. T., Fischer, A. H., \& Van Kleef, G. A. (2012). Face the noise: Embodied responses to nonverbal vocalizations of discrete emotions. Journal of Personality and Social Psychology, 102, 796-814. https://doi.org/10.1037/a0026234

Hawk, S. T., van Kleef, G. A., Fischer, A. H., \& van der Schalk, J. (2009). “Worth a thousand words": Absolute and relative decoding of nonlinguistic affect vocalizations. Emotion, 9, 293-305. https://doi.org/10.1037/a0015178

Hess U. (2021). Who to whom and why: The social nature of emotional mimicry. Psychophysiology, 58, e13675. https://doi.org/10.1111/psyp.13675

Hess, U., \& Blairy, S. (2001). Facial mimicry and emotional contagion to dynamic emotional facial expressions and their influence on decoding accuracy. International Journal of Psychophysiology, 40, 129-141. https://doi.org/10.1016/s0167-8760(00)00161-6

Hess, U., \& Fischer, A. (2013). Emotional mimicry as social regulation. Personality and Social Psychology Review, 17, 142-157. https://doi.org/10.1177/1088868312472607b 
Hess, U., \& Fischer, A. (2014). Emotional mimicry: Why and when we mimic emotions. Social and Personality Psychology Compass, 8, 45-57. https://doi.org/10.1111/spc3.12083

Hess, U., \& Kleck, R. E. (1994). The cues decoders use in attempting to differentiate emotion-elicited and posed facial expressions. European Journal of Social Psychology, 24, 367-381. https://doi.org/10.1002/ejsp.2420240306

Hietanen, J. K. \& Peltola, M. J. (2021) The eye contact smile: The effects of sending and receiving a direct gaze. Visual Cognition. https://doi.org/10.1080/13506285.2021.1915904

Hietanen, J. K., Surakka, V., \& Linnankoski, I. (1998). Facial electromyographic responses to vocal affect expressions. Psychophysiology, 35, 530-536. DOI: https://doi.org/10.1017/s0048577298970445

Hietanen, J. K., Kylliäinen, A., \& Peltola, M. J. (2019). The effect of being watched on facial EMG and autonomic activity in response to another individual's facial expressions. Scientific Reports, 9, 14759. https://doi.org/10.1038/s41598-019-51368-6

Hietanen, J. O., Peltola, M. J., \& Hietanen, J. K. (2020). Psychophysiological responses to eye contact in a live interaction and in video call. Psychophysiology, 57, e13587. https://doi.org/10.1111/psyp.13587

Holland, A. C., O’Connell, G., Dziobek, I. (2021) Facial mimicry, empathy, and emotion recognition: A meta-analysis of correlations. Cognition and Emotion, 35, 150-168. https://doi.org/10.1080/02699931.2020.1815655

Israelashvili, J., Hassin, R. R., \& Aviezer, H. (2019). When emotions run high: A critical role for context in the unfolding of dynamic, real-life facial affect. Emotion, 19, 558-562. https://doi.org/10.1037/emo0000441 
Jarick, M., \& Bencic, R. (2019). Eye contact is a two-way street: Arousal is elicited by the sending and receiving of eye gaze information. Frontiers in Psychology, 10, 1262. https://doi.org/10.3389/fpsyg.2019.01262

Juslin, P. N., \& Laukka, P. (2003). Communication of emotions in vocal expression and music performance: different channels, same code? Psychological Bulletin, 129, 770814. https://doi.org/10.1037/0033-2909.129.5.770

Kämpf, S. M., Liebermann, H., Kerschreiter, R., Krause, S., Nestler, S., \& Schmukle, S. C. (2018). Disentangling the sources of mimicry: Social relations analyses of the link between mimicry and liking. Psychological Science, 29, 131-138. https://doi.org/10.1177/0956797617727121

Kret, M. E., Fischer, A., H., \& De Dreu, C. K. W. (2015). Pupil mimicry correlates with trust in in-group partners with dilating pupils. Psychological Science, 26, 1401-1410. https://doi.org/10.1177/0956797615588306

Krumhuber, E. G., Küster, D., Namba, S., Shah, D., \& Calvo, M. G. (2021). Emotion recognition from posed and spontaneous dynamic expressions: Human observers versus machine analysis. Emotion, 21, 447-451. https://doi.org/10.1037/emo0000712

Kuang, B., Li, X., Li, X., Lin, M., Liu, S., \& Hu, P. (2021). The effect of eye gaze direction on emotional mimicry: A multimodal study with electromyography and electroencephalography. NeuroImage, 226, 117604.

https://doi.org/10.1016/j.neuroimage.2020.117604

Lakin, J. L., Jefferis, V. E., Cheng, C. M., \& Chartrand, T. L. (2003). The chameleon effect as social glue: Evidence for the evolutionary significance of nonconscious mimicry. Journal of Nonverbal Behavior, 27, 145-162.

https://doi.org/10.1023/A:1025389814290 
Leng, Y., Zhu, Y., Ge, S., Qian, X., \& Zhang, J. (2018). Neural Temporal Dynamics of Social Exclusion Elicited by Averted Gaze: An Event-Related Potentials Study. Frontiers in Behavioral Neuroscience, 12, 21. https://doi.org/10.3389/fnbeh.2018.00021

Likowski, K. U., Mühlberger, A., Seibt, B., Pauli, P., \& Weyers, P. (2008). Modulation of facial mimicry by attitudes. Journal of Experimental Social Psychology, 44, 1065-1072. https://doi.org/10.1016/j.jesp.2007.10.007

Lima, C. F., Arriaga, P., Anikin, A., Pires, A. R., Frade, S., Neves, L., \& Scott, S. K. (2021). Authentic and posed emotional vocalizations trigger distinct facial responses. Cortex, 141, 280-292. https://doi.org/10.1016/j.cortex.2021.04.015

Lischetzke, T., Cugialy, M., Apt, T., Eid, M., \& Niedeggen, M. (2019). Are those who tend to mimic facial expressions especially vulnerable to emotional contagion? Journal of Nonverbal Behavior, 44, 133-152. https://doi.org/10.1007/s10919-019-00316-Z

Magnée, M. J. C. M., Stekelenburg, J. J., Kemner, C., \& de Gelder, B. (2007). Similar facial electromyographic responses to faces, voices, and body expressions. NeuroReport, 18, 369-372. https://doi.org/10.1097/WNR.0b013e32801776e6

Marschner, L., Pannasch, S., Schulz, J., \& Graupner, S. T. (2015). Social communication with virtual agents: The effects of body and gaze direction on attention and emotional responding in human observers. International Journal of Psychophysiology, 97, 85-92. https://doi.org/10.1016/j.ijpsycho.2015.05.007

Mason, M. F., Tatkow, E. P., \& Macrae, C. N. (2005). The look of love: gaze shifts and person perception. Psychological Science, 16, 236-239. https://doi.org/10.1111/j.09567976.2005.00809.x

Myllyneva, A., \& Hietanen, J. K. (2015). There is more to eye contact than meets the eye. Cognition, 134, 100-109. https://doi.org/10.1016/j.cognition.2014.09.011 
Neumann, R., \& Strack, F. (2000). "Mood contagion": The automatic transfer of mood between persons. Journal of Personality and Social Psychology, 79, 211-223. https://doi.org/10.1037/0022-3514.79.2.211

Niedenthal, P. M. (2007). Embodying emotion. Science, 316, 1002-1005. https://doi.org/10.1126/science. 1136930

Neidenthal, P. M., Brauer, M., Halberstadt, J. B., \& Innes-Ker, Å. H. (2001). When did her smile drop? Facial mimicry and the influences of emotional state on the detection of change in emotional expression. Cognition and Emotion, 15, 853-864.

https://doi.org/10.1080/02699930143000194

Niedenthal, P. M., Barsalou, L. W., Winkielman, P., Krauth-Gruber, S., \& Ric, F. (2005). Embodiment in attitudes, social perception, and emotion. Personality and Social Psychology Review, 9, 184-211. https://doi.org/10.1207/s15327957pspr0903_1

Niedenthal, P. M., Mermillod, M., Maringer, M., \& Hess, U. (2010). The Simulation of Smiles (SIMS) model: Embodied simulation and the meaning of facial expression. Behavioral and Brain Sciences, 33, 417-480.

\section{https://doi.org/10.1017/S0140525X10000865}

Neal, D. T., \& Chartrand, T. L. (2011). Embodied emotion perception: Amplifying and dampening facial feedback modulates emotion perception accuracy. Social Psychological and Personality Science, 2, 673-678.

https://doi.org/10.1177/1948550611406138

Niedenthal, P. M., Wood, A., Rychlowska, M., \& Korb, S. (2017). Embodied simulation in decoding facial expression. In J.-M. Fernández-Dols \& J. A. Russell (Eds.), The science of facial expression (pp. 397-413). Oxford University Press.

https://doi.org/10.1093/acprof:oso/9780190613501.003.0021 
Oberman, L. M., Winkielman, P. \& Ramachandran, V. S. (2007). Face to face: Blocking facial mimicry can selectively impair recognition of emotional expressions. Social Neuroscience, 2, 167-178. https://doi.org/10.1080/1747091070139194

Oh Kruzic, C., Kruzic, D., Herrera, F., \& Bailenson, J. (2020). Facial expressions contribute more than body movements to conversational outcomes in avatar-mediated virtual environments. Scientific Reports, 10, 20626. https://doi.org/10.1038/s41598-020-76672$\underline{4}$

Olszanowski, M., Pochwatko, G., Kuklinski, K., Scibor-Rylski, M., Lewinski, P., \& Ohme, R. K. (2015). Warsaw set of emotional facial expression pictures: A validation study of facial display photographs. Frontiers in Psychology, 5, 1516. https://doi.org/10.3389/fpsyg.2014.01516

Olszanowski, M., Wróbel, M., \& Hess, U. (2020). Mimicking and sharing emotions: a reexamination of the link between facial mimicry and emotional contagion. Cognition \& Emotion, 34, 367-376. https://doi.org/10.1080/02699931.2019.1611543

Orlowska, A., Rychlowska, M., Szarota, P., \& Krumhuber, E. (2021, June 11). Facial mimicry and social context affect smile interpretation. Retrieved from https://doi.org/10.31234/osf.io/34e5k

Ortony, A., \& Turner, T. J. (1990). What's basic about basic emotions? Psychological Review, 97, 315-331. https://doi.org/10.1037/0033-295X.97.3.315

Paulmann, S., \& Pell, M. D. (2011). Is there an advantage for recognizing multi-modal emotional stimuli? Motivation and Emotion, 35, 192-201. https://doi.org/10.1007/s11031-011-9206-0

Rueff-Lopes, R., Navarro, J., Caetano, A., \& Silva, A. J. (2015). A Markov chain analysis of emotional exchange in voice-to-voice communication: Testing for the mimicry 
hypothesis of emotional Contagion. Human Communication Research, 41, 412-434. https://doi.org/10.1111/hcre.12051

Rychlowska, M., Zinner, L., Musca, S. C., \& Niedenthal, P. M. (2012). From the eye to the heart eye contact triggers emotion simulation. Proceedings of the 4th Workshop on Eye Gaze in Intelligent Human Machine Interaction, 5, 1-17 https://doi.org/10.1145/2401836.2401841

Schrammel, F., Pannasch, S., Graupner, S. T., Mojzisch, A., \& Velichkovsky, B. M. (2009). Virtual friend or threat? The effects of facial expression and gaze interaction on psychophysiological responses and emotional experience. Psychophysiology, 46, 922 931. https://doi.org/10.1111/j.1469-8986.2009.00831.x

Soussignan, R., Chadwick, M., Philip, L., Conty, L., Dezecache, G., \& Grèzes, J. (2013). Self-relevance appraisal of gaze direction and dynamic facial expressions: effects on facial electromyographic and autonomic reactions. Emotion, 13, 330-337. https://doi.org/10.1037/a0029892

Van der Schalk, J., Fischer, A., Doosje, B., Wigboldus, D., Hawk, S., Rotteveel, M., \& Hess, U. (2011). Convergent and divergent responses to emotional displays of ingroup and outgroup. Emotion, 11, 286-298. https://doi.org/10.1037/a0022582

van Kleef, G. A. (2017). Emotions as agents of social influence: Insights from Emotions as Social Information (EASI) theory. In S. G. Harkins, K. D. Williams, \& J. M. Burger (Eds.), The Oxford Handbook of Social Influence (pp. 237-255). Oxford University Press. https://doi.org/10.1093/oxfordhb/9780199859870.013.19

Wang, Y., \& Hamilton, A. F. (2014). Why does gaze enhance mimicry? Placing gazemimicry effects in relation to other gaze phenomena. Quarterly Journal of Experimental Psychology, 67, 747-762. https://doi.org/10.1080/17470218.2013.828316 
Wang, Y., Newport, R., \& Hamilton, A. F. (2011). Eye contact enhances mimicry of intransitive hand movements. Biology Letters, 7, 7-10. https://doi.org/10.1098/rsbl.2010.0279

Winkielman, P., Coulson, S., \& Niedenthal, P. (2018). Dynamic grounding of emotion concepts. Philosophical transactions of the Royal Society of London. Series B, Biological Sciences, 373, 20170127. https://doi.org/10.1098/rstb.2017.0127

Wróbel, M., Imbir, K. (2019). Broadening the perspective on emotional contagion and emotional mimicry: The correction hypothesis. Perspectives on Psychological Science, 14, 437-451. https://doi.org/10.1177/1745691618808523 